\title{
COMPARISON OF DENTINAL TUBULAR PENETRATION OF THREE BIOCERAMIC SEALERS
}

\author{
CELINE MARISSA $^{1}$, MUNYATI USMAN ${ }^{2}$, ENDANG SUPRASTIWI ${ }^{2}$, ANITA ERDIANI $^{1}$, RATNA MEIDYAWATI ${ }^{2 *}$
}

${ }^{1}$ Conservative Dentistry Residency Program, Faculty of Dentistry, Universitas Indonesia. ${ }^{2}$ Department of Conservative Dentistry, Faculty of Dentistry, Universitas Indonesia. Email: meidyawati58@gmail.com

Received: 30 July 2019, Revised and Accepted: 01 June 2020

ABSTRACT

Objective: The main objective of endodontic treatment is to remove microorganisms from the root canal space and prevents reinfection. Deep penetration of the dentinal tubule is advantageous because it increases the contact area between the root canal filling material and dentin, thereby increasing the sealing quality of the entire root canal system. Bioceramic sealers are biocompatible, nontoxic, non-shrinking, hydrophilic, and stable, do not expand during setting, and can form hydroxyapatite, which then forms chemical bonds with dentin to compare the abilities of three types of bioceramic-based sealers to penetrate the dentinal tubules.

Methods: Obturation used three types of bioceramic sealers. Group 1 (calcium phosphate silicate), Group 2 (a mixture of tricalcium silicate and resin), and Group 3 (pure tricalcium silicate) were observed using a scanning electron microscope and measurement of the penetration distance with ImageJ.

Results: The bioceramic sealers had statistically significant differences in penetration distance into dentinal tubules (p's $<0.001$ ). The mean penetration value of Group 1 (calcium phosphate silicate) was $115.99 \mu \mathrm{m}$, Group 2 (a mixture of tricalcium silicate and resin) was $209.28 \mu \mathrm{m}$, and Group 3 (pure tricalcium silicate) was $84.07 \mu \mathrm{m}$.

Conclusion: Although all three bioceramic sealers penetrated the dentinal tubules, they exhibited differences in their penetration capabilities. Group 2 had the deepest penetration, likely due to the resin content.

Keywords: Penetration, Sealers, Bioceramics, Dentinal tubules, Scanning electron microscope, Calcium phosphate Silicate sealer, Tricalcium silicate sealer, Resin sealer.

(C) 2020 The Authors. Published by Innovare Academic Sciences Pvt Ltd. This is an open access article under the CC BY license (http://creativecommons. org/licenses/by/4. 0/) DOI: http://dx.doi.org/10.22159/ijap.2020.v12s2.0P-05

\section{INTRODUCTION}

The main goals of root canal treatment are to eliminate root canal microorganisms and to prevent root canal reinfection following treatment [1]. In endodontics, a hermetic root canal filling must be obtained using a sealer between the dentin wall and gutta-percha, a requirement referred to as the "monoblock" concept. "Monoblock" indicates the creation of a strong, solid, and comprehensive root canal between the material and root canal wall to prevent bacteria from entering the root canal system [2].

Gutmann stated that, along with the removal of debris and bacteria, good adaptation to the dentinal wall is essential to achieve an optimal root canal filling. This can be seen from the penetration of the material into the dentinal tubules [3]. Deep penetration of dentinal tubules is advantageous because it increases the contact area between the root canal filling material and dentin, thereby increasing the sealing quality of the entire root canal system [4]. The sealer penetration depth depends on many factors, including smear layer cleanliness, dentin permeability, root canal dimensions, and the physical and chemical properties of the sealer (flow rate) [5].

Bioceramics containing calcium phosphate silicate sealers were introduced in 2007. This sealer contains calcium silicates and calcium phosphate, which produces calcium ions to provide good bioactive biocompatibility. In 2010, the dental company Angelus issued a bioceramic sealer containing tricalcium silicate, dicalcium silicate, calcium oxide, tricalcium aluminate, resin, and bismuth oxide. The presence of tricalcium silicate is expected to provide better results in regeneration [6]. In 2015, Septodont issued the latest bioceramic sealer made with active biosilicate technology that contains pure tricalcium silicate, zirconium oxide, and calcium chloride [7]. This sealer is recommended for single-cone filling techniques; the application procedure is fast and easy, allowing this bioceramic to become a useful alternative filling technique [8].

The purpose of the present study was to analyze how bioceramic sealer composition affects the depth of penetration in dentinal tubules and sealing ability by comparing these three types of bioceramic sealers

\section{METHODS}

The selected extracted samples used in this study were 27 mandibular premolars (Malay race, extracted for orthodontic treatment in University of Indonesia) with single and straight root canals for easier preparation. This tooth can be easily obtained because it is often indicated for orthodontic treatment. All specimens were cleaned using a scaler then soaked in $0.9 \% \mathrm{NaCl}$ solution (OneMed, Indonesia), until the preparation and root canal filling were performed. The extracted teeth were prepared for access with round diamond burs until straight access was obtained into the root canal (ethical clearance no. 051111018).

The operator prepared the samples following the crown-down technique using a Rotary ProTaper Next instrument (Dentsply, Switzerland) until X3. For root canal preparation, $17 \%$ EDTA gel (RC-Prep ${ }^{\circledR}$ Premiere, USA) was used as a lubricant when changing instruments. The root canal then irrigated with $2 \mathrm{ml}$ of $2.5 \% \mathrm{NaOCl}$ (OneMed, Indonesia) and activated with sonic instruments (Endoactivator, Dentsply). Each instrument was rinsed with $2 \mathrm{ml}$ distilled water solution when changed. After root canal preparation, all root canals were irrigated with $17 \%$ EDTA solution, left for $1 \mathrm{~min}$, then rinsed with $2 \mathrm{ml}$ distilled water.

The obturation process used gutta-percha and one of three bioceramic sealers. The 27 samples were divided into three groups (n's=9) of bioceramic sealers: Group 1 used calcium phosphate silicate (IRoot ${ }^{\circledR} \mathrm{Sp}$, 
Innovative BioCeramix, Inc., Vancouver, Canada), Group 2 was a mixture of tricalcium silicate and resin (MTA Fillapex, Angelus, Brazil), and Group 3 was pure tricalcium silicate (BioRoot RCS, Septodont, France). The working length was set as $\pm 0.5 \mathrm{~mm}$ from the apical foramen. Each root canal was prepared, irrigated, and dried with a paper point before bioceramic sealer was applied according to the manufacturer's instructions. The main gutta-percha cone (ProTaper Next) was then smeared with sealer and inserted into the root canal. The excess sealer was removed, and the cones were cut using hot instruments. The coronal section was sealed with resin-modified glass ionomer cement. Next, the quality of the root canal filling was evaluated using Digora digital radiography (Soredex, Finland). Specimens were stored in an incubator at $37^{\circ} \mathrm{C}$ with $100 \%$ humidity for 3 days to thoroughly set the sealer.

After 3 days, the samples were cut by the operator at $5 \mathrm{~mm}$ from the apex, mounted in acrylic, polished, and gold coated. Subsequently, the samples were observed using a scanning electron microscope (SEM). The penetration distance of the sealer into the dentinal tubules was collected and calculated using ImageJ software.

Data regarding the penetration distance of the sealer material into the tubular root canal dentin were analyzed using SPSS software version 22.0. Shapiro-Wilk normality tests indicated that all data were parametric $(\mathrm{p}>0.05)$. Therefore, one-way ANOVA statistical tests were conducted with multiple comparison post hoc tests. Significance was set at $\mathrm{p}<0.05$.

\section{RESULTS}

In this study, three groups of nine teeth were obturated using singlecone techniques and a bioceramic sealer. The sealer used for the first group contained calcium phosphate silicate (IRoot ${ }^{\circledR}$ SP); the second group sealer contained a mixture of tricalcium silicate and resin (MTA Fillape $\left.{ }^{\circledR}\right)$; and the third group contained pure tricalcium silicate (BioRoot ${ }^{\text {Tm }}$ RCS). The results of the filling were evaluated using a digital radiograph (Soredex, Finland). The samples were observed using a field emission scanning electron microscope, with measurements made using ImageJ software.

Although all three bioceramic sealers penetrated the dentin tubules, a one-way ANOVA $(\mathrm{F}=131.019, \mathrm{p}=0.001)$ revealed that they did so to significantly different depths (Table 1). Tamhane post hoc analyses revealed that Group 2 had significantly higher penetration than Groups 1 and 3, whereas Group 3 had significantly lower penetrations than Groups 1 and $2(\mathrm{p}<0.05)$.

\section{DISCUSSION}

The main purpose of root canal obturation is to close the communication pathway between the root canal system and periradicular tissue by creating a barrier to prevent reinfection. In root canal obturation, a monoblock bond is expected to be created among the gutta-percha, sealer, and root canal wall dentin. Here, the abilities of three different bioceramic sealers to penetrate into the dentinal tubules were compared. The sealers compared contained calcium silicate phosphate, a mixture of tricalcium silicate and pure resin, and tricalcium silicate [3].

Table 1: Mean value and standard deviation of the dentinal tubule penetration distance of three bioceramic sealer types. p-values indicate significance values representing post hoc group differences

\begin{tabular}{llll}
\hline Group & n (teeth) & Mean (SD) & p-value \\
\hline Group 1 & 9 & $115.99(13.1)$ & 0.001 \\
Group 2 & 9 & $209.28(34.31)$ & 0.001 \\
Group 3 & 9 & $84.07(5.6)$ & 0.001 \\
\hline
\end{tabular}

Group 1: Bioceramic sealer containing calcium phosphate silicate,

Group 2: Bioceramic sealer containing a mixture of tricalcium silicate and resin,

Group 3: Bioceramic sealer containing pure tricalcium silicate
Sealer is important for filling the irregular space and adhering root canal accessories. Sealer penetration into the canal tubules increases surface contact between the filling material and dentin. A wider contact surface allows for greater sealing ability to prevent bacterial penetration. Bacterial penetration of the dentinal tubules can reach 100-1000 $\mu \mathrm{m}$ and can increase with the loss of the smear layer. Bacteria that deeply penetrate the dentinal tubules are typically of the facultative anaerobic species. The sealer can trap the remaining bacteria in the dentinal tubules and deactivate them. This plays an important role in the periapical lesion healing process. Thus, the dentinal tubule penetration ability of sealers is an important factor when choosing the type of sealer [4].

The sealer penetration depth depends on many factors, including smear layer elimination, dentin permeability (tubule diameter and diameter), root canal dimensions, and the physical and chemical properties of the sealer (flow rate) [6]. The sealer flow rate is determined by composition, particle size, film thickness, and consistency of ingredients. Most sealers are pseudoplastic with a low viscosity that enables them to flow into the dentinal tubules and root canal accessories.

This current study used bioceramic sealer due to its good physicochemical and biocompatibility properties. It has low solubility and is dimensionally stable over a long period of time [8-10]. Bioceramic sealer bonds to the dentin wall and forms hydroxyapatite. According to Sagsen et al. [11], bioceramic sealer that contains calcium phosphate silicate forms a better bond than bioceramic sealer containing a mixture of tricalcium silicate and resin. Ghoneim et al. [12] reported that bioceramic silicones are containing calcium phosphate silicate increase tooth fracture resistance following root canal treatment in vitro, which may be related to the high bond strength of this sealer.

The current study observed that the area $5 \mathrm{~mm}$ from the apical are because accessory and lateral root canals often lead to ramifications in this area, which may affect the pathways of communication to the periodontal tissue. Hermetic sealing in the $1 / 3$ apical area is important to prevent reinfection of the root canal system (Fig. 1) [13].

The SEM observation of each group tubuli dentin penetration can be seen through (Figs. 2-4). The results of the comparative analysis of the penetration distances of the three types of bioceramic sealer into the dentinal tubules indicated that all the sealers had the ability to penetrate the dentinal tubules. This is due to the good physicochemical properties of these sealers [14]

According to Giudice et al. (2015), dentin is a hard tissue that forms the main part of the tooth. It consists of type I collagen fibrils (and small amounts of type III, collagen IV, non-collagen proteins, and proteoglycans) and hydroxylapatite [15].

Sealer penetration into the dentinal tubules can also be determined by the diameter of the dentinal tubules and particle size of the sealer. The diameter of the sealer particles must be smaller than that of the tubules to penetrate deeply. In the SEM analysis, the particles in Group 3 were in the form of granules; contained carbon, nitrogen, oxygen, calcium, and zirconium; and had a diameter of 2-10 $\mu \mathrm{m}$. AlHaddad et al. observed that bioceramic sealers containing a mixture of tricalcium silicate and resin primarily exhibited salicylate resin, diluting resin, and natural resin, bismuth trioxide, silica nanoparticle, $40 \%$ MTA (tricalcium silicate), and pigment as their largest components [6]. However, other bioceramic sealers that contain calcium phosphate silicate primarily show zirconium oxide, calcium silicates, calcium phosphate, calcium hydroxide, sealer, and thickening agents as their largest components [15].

The current study revealed that the Group 2 bioceramic sealer (containing a mixture of tricalcium silicate with resin) had the highest penetration depth into the dentinal tubules, followed by Group 1 (calcium bioceramic silicate), then Group 3 (pure tricalcium 
silicate). This might be due to the natural resin properties contained in these sealers. Although bioceramic sealers that contain a mixture of tricalcium silicate and resin are typically referred to as calcium silicatebased, the composition is dominated by resin, especially salicylate resin. The penetration depth of these sealers into the dentinal tubules has not been evaluated. Silva et al. (2013) compared the flow of bioceramic sealer containing a mixture of tricalcium silicate and epoxy resin-based sealers, showing that this sealer had a higher flow (31 mm) than epoxy resin-based sealers (26 mm) [16]. Abada et al. (2015) found that bioceramic sealers that contained a mixture of tricalcium silicate and resin had a higher flow than those containing calcium phosphate silicate and pure tricalcium silicate $(29.04,26.96$, and around $26 \mathrm{~mm}$, respectively). However, it is unknown whether this remains true when sealers are inserted into root canals in vivo [17].

Flow rate is also determined by sealer consistency. The consistency of the Group 2 sealer (tricalcium silicate and resin) was more fluid than the other two sealers. This is in accordance with the Washburn equation, which indicates that the sealer will flow more easily into the dentinal tubules due to capillary force. In Group 3 (pure tricalcium silicate), the sealer consistency was like paste, with a higher viscosity, resulting in the lowest penetration distance [16].

According to Tuncer et al., liquid-shaped resins with smaller particle sizes allow for easier flow into the dentinal tubules [18,19]. Siboni et al. suggested that, because bioceramic sealer containing calcium phosphate silicate has very small particles $(<1 \mu \mathrm{m})$ that are hydrophilic and have a low contact angle, the sealer can spread into lateral root canals

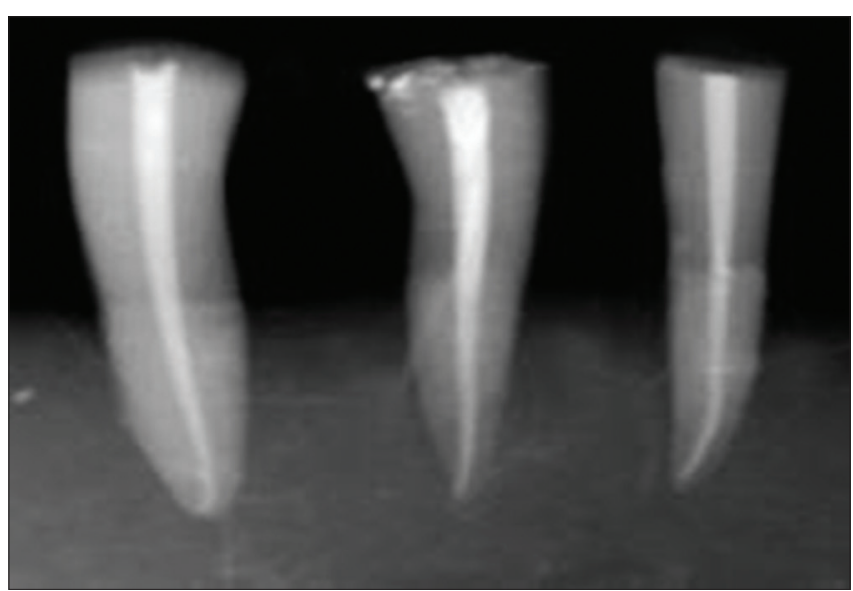

Fig. 1: Filling evaluation using digital radiography. A. Group 1 (bioceramic sealer containing calcium phosphate silicate).

B. Group 2 (bioceramic sealer containing a mixture of tricalcium silicate and resin). C. Group 3 (bioceramic sealer containing pure tricalcium silicate)
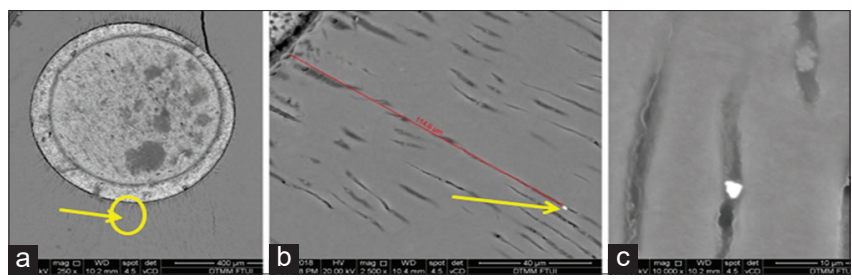

Fig. 2: Scanning electron microscope observation of Group 1 (bioceramic sealer containing calcium phosphate silicate). A. At a magnification of $\times 250$, there was bonding among the sealer gutta-percha, and dentin wall. The sealer and gutta-percha

bonded completely, but a gap still existed between the sealer and dentin wall. $B$. The furthest penetration distance $(\times 2500)$.

C. Dentin tubules filled with bioceramic sealer containing calcium phosphate silicate $(\times \mathbf{1 0 0 0 0})$ and accessories, allowing for good penetration ability. Therefore, the significant difference in penetration distance between the bioceramic sealer containing pure tricalcium silicate and the other two types of sealer in the current study may be due to the larger size of the sealer particles $(2-10 \mu \mathrm{m})$ compared with the diameter of the dentin tubule at the apical region $(1-2 \mu \mathrm{m})[20]$.

Another factor that affects the flow rate is film thickness, with thinner material flowing more easily into the dentinal tubules. Zhou et al. reported that epoxy resin-based sealers have a higher flow rate due to their thinner film thickness $(16.07 \mu \mathrm{m})$ compared with bioceramic films. However, there was no significant difference in film thickness between bioceramic sealers containing calcium silicate phosphate $(22 \mu \mathrm{m})$ and those containing a mixture of tricalcium silicate with resin $(23.92 \mu \mathrm{m})$ [9]. According to Camilleri et al., bioceramic sealer containing pure tricalcium silicate has a greater maximum film thickness $(45 \mu \mathrm{m})$ than epoxy resin-based sealers. This is in line with the results of the current study, wherein Group 3 had the lowest penetration [21].

Theoretically, greater sealer penetration the dentinal tubules could improve mechanical retention by forming a plug and increasing the root canal filling sealing ability [18]. However, De-Deus et al. reported no significant correlation between sealing ability and sealer penetration into the dentinal tubules [22]. Tuncer et al. found that micromechanical retention caused by tag sealer penetration into the dentinal tubules was not a significant factor in root canal filling adhesion. Adhesion depends on several factors, including the surface of the adherend (dentin or gutta-percha), surface tension of the sealer, root canal moisture, and magnitude of the undersurface gap [18].

Tuncer et al. found that, although bioceramic sealer containing a mixture of tricalcium silicate and resin has the highest penetration depth, it did not necessarily bond best to dentinal tubules. This is due to
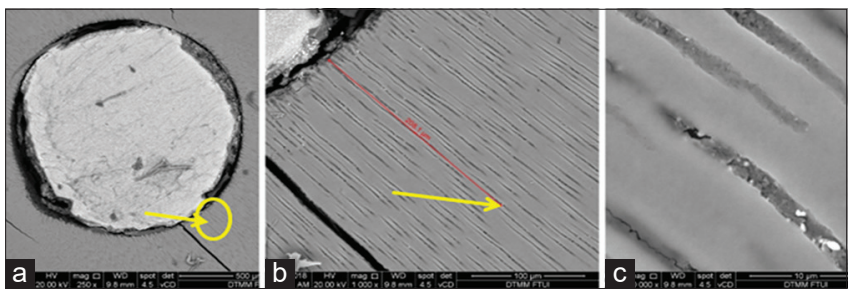

Fig. 3: Scanning electron microscope observation of Group 2 (bioceramic sealer containing tricalcium silicate and resin). A. At a magnification of $\times 250$, there was bonding among the sealer, gutta-percha, and dentin wall. The sealer bonded with the gutta-percha, but a large gap existed between the sealer and dentin wall. $B$. The furthest penetration distance $(\times 2500)$. C. Dentin tubules filled with sealer $(\times 10000)$
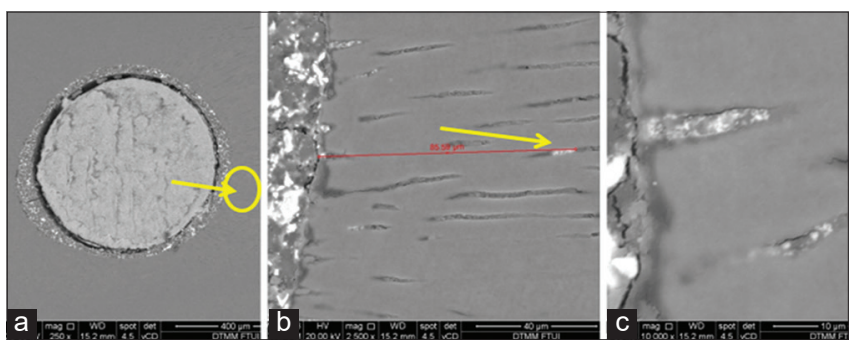

Fig. 4: Scanning electron microscope observation of Group 3 (bioceramic sealer containing pure tricalcium silicate). A. At a magnification of $\times 250$, there was bonding among the sealer, gutta-percha, and dentin wall. A gap existed between the guttapercha and sealer, but the sealer adapted well to the dentin wall. B. The furthest penetration distance $(\times 2500)$. C. Dentin tubulars filled with sealer $(\times 10000)$ 
the sealer's low bond strength and inability to form tag-like structures. Conversely, the high bond strength of calcium phosphate silicate sealers is due to the composition of calcium silicate, which does not allow any shrinkage during the setting process [18].

Thus, the current and previous studies indicate that the Group 2 bioceramic sealer shows the highest penetration due to its physiochemical properties of high flow rate, small particle size, and good consistency. However, these properties did not necessarily create the best monoblock bond. The resin contained in the sealer results in greater shrinkage than the other two sealers. Therefore, high penetration does not necessarily create a good dentin bond.

The bioceramic sealers used herein have advantages and disadvantages. Notably, none of them were able to form a monoblock bond among the gutta-percha, sealer, and dentin wall. Apart from penetration into the dentinal tubules, bonding of the sealer adhesion is also crucial for a monoblock bond. The composition of the calcium silicate and calcium phosphate sealers causes higher biomineralization to form hydroxyapatite, resulting in a $0.2 \%$ expansion. The sealer containing a mixture of tricalcium silicate and resin produces a micromechanical interlocking between the root canal wall and the sealer. This results in a better adhesion bond to the root canal wall, thereby minimizing microleakage.

\section{CONCLUSION}

The three bioceramic sealers investigated herein were able to penetrate the dentinal tubules, but to significantly different depths. The bioceramic sealer that contained a mixture of tricalcium silicate and resin exhibited the best penetration. In part, this is due to a higher flow rate that is enabled by the resin content, which has a liquid consistency, smaller particle size, and thinner film thickness.

\section{ACKNOWLEDGMENTS}

This study was supported by the Directorate of Research and Community Engagement of Universitas Indonesia. The publication of this manuscript was supported by Universitas Indonesia.

\section{REFERENCES}

1. Chivian N. Resilon--the missing link in sealing the root canal. Compend Contin Educ Dent 2004;25:823.

2. Singh H, Markan S, Kaur M, Gupta G, Singh H, Kaur MS. Endodontic sealers: Current concepts and comparative analysis. Dent Open J 2015;2:32-7.

3. Gutmann JL. Adaptation of injected thermoplasticized gutta-percha in the absence of the dentinal smear layer. Int Endod J 1993;26:87-92.

4. Chen H, Zhao X, Qiu Y, Xu D, Cui L, Wu B. The tubular penetration depth and adaption of four sealers: A scanning electron microscopic study. Biomed Res Int 2017;2017:2946524.

5. Balguerie E, Van der Sluis L, Vallaeys K, Gurgel-Georgelin M, Diemer F. Sealer penetration and adaptation in the dentinal tubules: A scanning electron microscopic study. J Endod 2011;37:1576-9.

6. Al-Haddad A, Ab Aziz C, Zeti A. Bioceramic-based root canal sealers: A review. Int J Biomater 2016;2016:9753210.

7. Camilleri J. BioRoot ${ }^{\mathrm{TM}}$ RCS. Endo Sealer or Biological Filler; 2018. Available from: https://www.septodontcorp.com.

8. Simon AC. BioRoot RCS A BIOACTIVE Breakthrough; 2018. Available from: https://www.septodontusa.com.

9. Zhou HM, Shen Y, Wang ZJ, Li L, Zheng YF, Häkkinen L, et al. In vitro cytotoxicity evaluation of a novel root repair material. J Endod 2013;39:478-83

10. Kokkas AB, Boutsioukis AC, Vassiliadis LP, Stavrianos CK. The influence of the smear layer on dentinal tubule penetration depth by three different root canal sealers: An in vitro study. J Endod 2004;30:100-2.

11. Sagsen B, Ustün Y, Demirbuga S, Pala K. Push-out bond strength of two new calcium silicate-based endodontic sealers to root canal dentine. Int Endod J 2011;44:1088-91.

12. Ghoneim AG, Lutfy RA, Sabet NE, Fayyad DM. Resistance to fracture of roots obturated with novel canal-filling systems. J Endod 2011;37:1590-2.

13. McMichael GE, Primus CM, Opperman LA. Dentinal tubule penetration of tricalcium silicate sealers. J Endod 2016;42:632-6.

14. Mamootil K, Messer HH. Penetration of dentinal tubules by endodontic sealer cements in extracted teeth and in vivo. Int Endod $\mathrm{J}$ 2007; 40:873-81.

15. Lo Giudice G, Cutroneo G, Centofanti A, Artemisia A, Bramanti E, Militi A, et al. Dentin morphology of root canal surface: A quantitative evaluation based on a scanning electronic microscopy study. BioMed Res Int 2015;2015:7.

16. Silva EJ, Rosa TP, Herrera DR, Jacinto RC, Gomes BP, Zaia AA. Evaluation of cytotoxicity and physicochemical properties of calcium silicate-based endodontic sealer siler biokeramik mengandung campuran trikalsium silikat dan resin. J Endod 2013;39:274-7.

17. Abada HM, Farag AM, Alhadainy HA, Darrag AM. Push-out bond strength of different root canal obturation systems to root canal dentin. Tanta Dent J 2015;12:185-91.

18. Chandra SS, Shankar P, Indira R. Depth of penetration of four resin sealers into radicular dentinal tubules: A confocal microscopic study. J Endod 2012;38:1412-6.

19. Tuncer AK, Tuncer S, Gökyay SS. Correlation between sealer penetration into dentinal tubules and bond strength of two new calcium silicate-based and an epoxy resin-based, endodontic sealer. J Adhes Sci Technol 2014;28:702-10.

20. Siboni F, Taddei P, Zamparini F, Prati C, Gandolfi MG. Properties of BioRoot RCS, a tricalcium silicate endodontic sealer modified with povidone and polycarboxylate. Int Endod J 2017;50:e120-36.

21. Camilleri J, Sorrentino F, Damidot D. Characterization of un-hydrated and hydrated BioAggregate ${ }^{\mathrm{TM}}$ and MTA Angelus ${ }^{\mathrm{TM}}$. Clin Oral Investig 2015;19:689-98

22. Leal F, De-Deus G, Brandao C, Luna A, Souza E, Fidel S. Similar sealability between bioceramic putty ready-to-use repair cement and white MTA. Braz Den J 2013;24:362-66. 